\title{
Metabonomics reveals peripheral and central short- chain fatty acid and amino acid dysfunction in a naturally occurring depressive model of macaques
}

This article was published in the following Dove Medical Press journal:

Neuropsychiatric Disease and Treatment

Feng-Li Deng, ${ }^{1-4, *}$ Jun-Xi Pan, 2,3,5,* Peng Zheng, 2,3,6 Jin-Jun Xia, ${ }^{2,3}$ Bang-Min Yin, ${ }^{1-3}$ Wei-Wei Liang, ${ }^{1-3}$ Yi-Fan Li, ${ }^{2,3,6}$ Jing $W u,{ }^{2,3}$ Fan $X u,{ }^{2,3}$ QingYuan Wu, 2,3,7 Chao-Hua Qu, 2,3 Wei Li, ${ }^{2,3}$ Hai-Yang Wang, ${ }^{2,3}$ Peng $\mathrm{Xie}^{1-3}$

'Department of Neurology, Yongchuan Hospital, Chongqing Medical University, Chongqing 402460, People's Republic of China; ${ }^{2}$ Chongqing Key Laboratory of Neurobiology, Chongqing 400016, People's Republic of China;

${ }^{3}$ Institute of Neuroscience and the Collaborative Innovation Center for Brain Science, Chongqing Medical University, Chongqing 400016, People's Republic of China; ${ }^{4}$ School of Public Health and Management, Chongqing Medical University, Chongqing 400016, People's Republic of China; ${ }^{5}$ The First Affiliated Hospital of Kunming Medical University, Kunming 650032, People's Republic of China; ' ${ }^{\circ}$ epartment of Neurology, The First Affiliated Hospital of Chongqing Medical University, Chongqing 4000 16, People's Republic of China; ${ }^{7}$ Department of Neurology, Three Gorges Central Hospital, Chongqing Medical University, Chongqing 4000I6, People's Republic of China

*These authors contributed equally to this work

Correspondence: Peng Xie

Department of Neurology, The First

Affiliated Hospital of Chongqing Medical

University, I Youyi Road, Yuzhong

District, Chongqing 4000I6, People's

Republic of China

Tel +862368485490

Fax +86 2368485 III

Email xiepeng@cqmu.edu.cn
Purpose: Depression is a complex psychiatric disorder. Various depressive rodent models are usually constructed based on different pathogenesis hypotheses.

Materials and methods: Herein, using our previously established naturally occurring depressive (NOD) model in a non-human primate (cynomolgus monkey, Macaca fascularis), we performed metabolomics analysis of cerebrospinal fluid (CSF) from NOD female macaques $(\mathrm{N}=10)$ and ageand gender-matched healthy controls $(\mathrm{HCs})(\mathrm{N}=12)$. Multivariate statistical analysis was used to identify the differentially expressed metabolites between the two groups. Ingenuity Pathways Analysis and MetaboAnalyst were applied for predicted pathways and biological functions analysis. Results: Totally, 37 metabolites responsible for discriminating the two groups were identified. The NOD macaques were mainly characterized by perturbations of fatty acid biosynthesis, ABC transport system, and amino acid metabolism (eg, aspartate, glycine, serine, and threonine metabolism). Interestingly, we found that eight altered CSF metabolites belonging to short-chain fatty acids and amino acids were also observed in the serum of NOD macaques ( $\mathrm{N}=13$ per group). Conclusion: Our findings suggest that peripheral and central short-chain fatty acids and amino acids are implicated in the onset of depression.

Keywords: naturally occurring depressive, depression, non-human primate, metabonomics, cerebrospinal fluid, short-chain fatty acid

\section{Introduction}

Depression is a serious mental illness worldwide, affecting more than 350 million people, equivalent to $4.4 \%$ of the world's population. ${ }^{1,2}$ Depression can be long-lasting or recurrent, substantially impairing not only patients' quality of life but also of their families. Moreover, depression can also lead to suicide, which is the second leading cause of death in adolescents. ${ }^{3,4}$ However, the underlying molecular mechanisms of depression remain largely unknown. ${ }^{5}$

Animal models are important tools for investigating the pathogenesis of depression. Our groups and other investigators established some rodent models to uncover the molecular mechanisms of depression..$^{3,5-9}$ These models play an important role in understanding the pathogenesis of depression. However, it cannot be overlooked that these models are based on some kind of pathogenesis theory. Under this condition, this may limit our horizons. To address this question, we recently had successfully screened naturally depressed monkeys in a similar natural cage, designated as a naturally occurring depressive (NOD) model of a non-human primate. Similar to depressive patients, the NOD monkeys exhibit depressed behavioral phenotype, such as low levels of activity, low levels of social interaction, and anhedonia. ${ }^{10}$ 
Cerebrospinal fluid (CSF) is arguably the most relevant sampling substrate for the in vivo study of brain disorders as it reflects the metabolic status and the biochemistry of the brain. ${ }^{11,12}$ Therefore, metabolomic profiles of CSF in patients and controls have the potential to reveal protein differences linked to the pathogenesis of neuropsychiatric disorder that might have value as biomarkers. ${ }^{13}$ Recent studies of CSF metabolite profiles have identified initial metabolic signatures for a variety of central nervous system (CNS) disorders, including depression, bipolar disorder, and Parkinson's disease. ${ }^{11,14,15}$ These metabolite profiles may potentially be useful as biomarkers in neurological conditions. However, as the clinical symptoms of depression are diverse and highly heterogeneous, it is valuable to perform the CSF analysis of NOD monkey model.

Here, a non-targeted metabolomics approach based on gas chromatography-mass spectrometry (GC-MS) was used to identify differentially expressed metabolites between NOD and control macaques based on a previous study. ${ }^{16}$ To systematically analyze the role of differentially expressed metabolites, the differential metabolites identified in this study were compared with those reported in our published findings. ${ }^{16}$ Some key metabolites were detected both in the and CSF, which may provide an evidence to understand peripheral and central interactions.

\section{Materials and methods Subjects and ethical statement}

All the cynomolgus monkeys were obtained from Xishan Zhongke Laboratory Animals Co. Ltd. (license number SYXK [Su 2002-0032). Animals were reared in socially stable colonies with negligible rates of conflict, were provided with water ad libitum, and fed daily with fresh fruits, vegetables, and high-nutrition monkey food. Each colony was housed in an indoor free enclosure measuring $12.0 \times 4.0 \times 3.0 \mathrm{~m}^{3}$ $(\mathrm{L} \times \mathrm{W} \times \mathrm{H})$ with 12-hour light/dark cycle (Figure 1A). Indoor pens were maintained at a temperature higher than $18^{\circ} \mathrm{C}$ and relative humidity of $40 \%-70 \%$. The living environment and animal care procedures are detailed in a previous report. ${ }^{17-20}$

All experiments in this study were approved by the Ethics Committee of Chongqing Medical University (approval no: 20100031), and all procedures were performed in accordance with the recommendations of the Weatherall report, "The use of non-human primates in research". ${ }^{21,22}$ Special care was taken to minimize the number and suffering in all experiments involving non-human primates. For more details, please refer to our previous publication..$^{16,20}$

\section{Behavioral observation and subject selection}

In our previous study, ${ }^{18}$ we systematically documented the comprehensive systematic ethogram of Macaca fascicularis
A

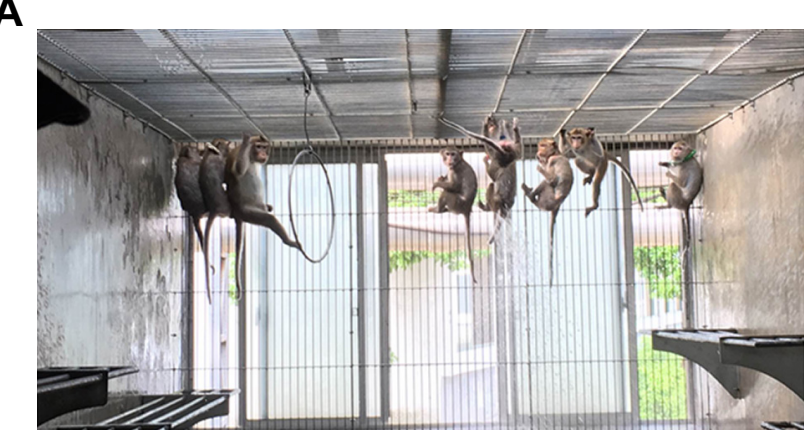

B
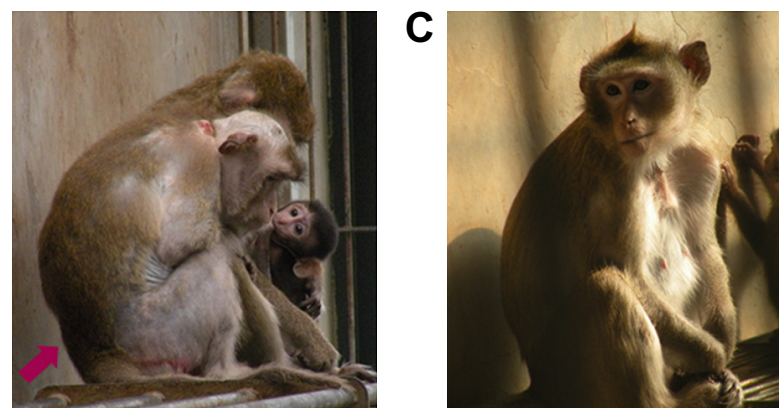

D

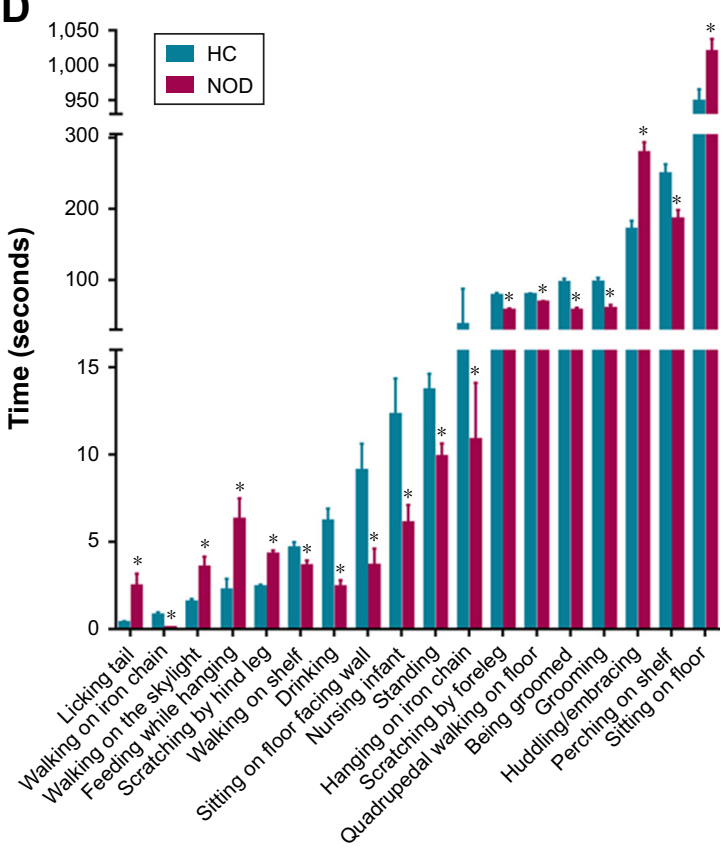

Figure I Behavioral observation of the cynomolgus monkeys. (A) Cynomolgus monkeys in a realistic social context. (B) Slumped or collapsed posture of a NOD macaque (red arrow). (C) A CON macaque. (D) The significant differential behavioral results between NOD macaques $(n=10)$ and CON macaques $(n=12)$.

Note: *Behaviors of greater duration in healthy controls relative to NOD macaques.

Abbreviations: CON, control; NOD, naturally occurring depressive; HC, healthy control. 
for behavioral investigations. We classified 53 discrete behaviors into 12 behavioral categories, which were applied in the present study (Table S1). All behavior patterns of each macaque were videotaped, and the three independent observers blindly and discretely scored all behavioral items on the videotape footage using NOLDUS Observer XT software (version 10.0; Noldus Information Tech Technology, Leesburg, PA, USA). ${ }^{16,18}$ Based on the behavioral observation, a NOD state of M. fascicularis was described..$^{18}$ The macaques ( $\mathrm{n}=13$ per group) that displayed the highest frequency of depressive behaviors over the observational period were selected in our previous study. ${ }^{16}$ Among the 13 NOD macaques, ten female macaques displayed the highest frequency of depressive behaviors and were selected for this study (Figure 1B). To match by age and sex, 12 of the 13 healthy female adult macaques were selected (controls, $\mathrm{CON}$ ) (Figure 1C). There was no significant difference in age or weight between the NOD macaques and CON group (Table 1, $P>0.05)$. Non-pregnant estrous cycles of female macaques were recorded, during which sample collection was avoided.

\section{Sample collection and preparation for GC-MS analysis}

CSF sampling was done as previously described ${ }^{23,24}$ Briefly, monkeys were removed from their home pens, and anesthetized by injecting $15 \mathrm{mg} / \mathrm{kg}$ ketamine intramuscularly. Sedation was achieved in $<5$ minutes after returning to their respective transfer cages. A $5 \mathrm{~cm}$ margin was shaved laterally to the midline on each side between lumbar vertebrae L5/6 for CSF collection. Under aseptic conditions, a needle (21-gauge, 0.0285 in) was used for CSF collection. About $1.5 \mathrm{~mL}$ of CSF was withdrawn, initially centrifuged at 2,000 rpm for 10 minutes at $4^{\circ} \mathrm{C}$, and divided into equal aliquots and stored at $-80^{\circ} \mathrm{C}$ until further use.

About $15 \mu \mathrm{L}$ of CSF sample was added to $10 \mu \mathrm{L}$ of L-leucine-13C6 $(0.1 \mathrm{mg} / \mathrm{mL})$ which acts as an internal standard. After vortexing for 30 seconds, $90 \mu \mathrm{L}$ of methanol was added. After centrifuging at $14,000 \mathrm{rpm}$ for 15 minutes, $95 \mu \mathrm{L}$ of supernatant was evaporated to dryness under a stream of nitrogen gas. The dried residue was added to $30 \mu \mathrm{L}$ of methoxamine hydrochloride $(20 \mathrm{mg} / \mathrm{mL}$ pyridine $)$ and incubated at $37^{\circ} \mathrm{C}$ for 90 minutes with continuous shaking. Subsequently, the solution was treated with $30 \mu \mathrm{L}$ of N,O-Bis(trimethylsilyl) trifluoroacetamide with $1 \%$ trimethylchlorosilane at $70^{\circ} \mathrm{C}$ for 60 minutes. After derivatization and cooling to room temperature, this derivative was injected in the GC-MS for analysis.

\section{GC-MS acquisition and data analysis}

GC-MS analysis was carried out according to this group's previously published work. ${ }^{5,16}$ Briefly, we used an Agilent7890A/5975C GC-MS System (Agilent Technologies Inc., Santa Clara, CA, USA) and a HP-5MS capillary column $(30 \mathrm{~m} \times 0.25 \mathrm{~mm} \times 0.25 \mu \mathrm{m}$; Agilent, Technologies Inc). Typical GC settings and MS parameters were used. Briefly, each $1 \mu \mathrm{L}$ of derivative sample was injected into the system with an injector temperature of $280^{\circ} \mathrm{C}$. Helium was used as a carrier gas at a constant flow rate of $6 \mathrm{~mL} / \mathrm{min}$. The column temperature was initially kept at $80^{\circ} \mathrm{C}$ for 2 minutes and then increased to $320^{\circ} \mathrm{C}$ gradually at the rate of $10^{\circ} \mathrm{C} / \mathrm{min}$, and maintained for 6 minutes. The column effluent was introduced into the ion source of mass selective detector. The MS quadrupole temperature was set at $150^{\circ} \mathrm{C}$, and the ion source temperature was set at $230^{\circ} \mathrm{C}$. Data acquisition was performed in the full-scan mode from 50 to $600 \mathrm{~m} / \mathrm{z}$. To avoid the influence induced by instrument signal fluctuations, a random order of continuous sample analysis was adopted.

The chromatographic peaks in the total ion current (TIC) chromatograms represent the corresponding metabolites, and their relative concentrations can be detected using the peak area normalization method. In the present study, the TIC from the CSF samples of two groups revealed strong signals for analysis, large peak capacity, and good retention time reproducibility (Figure 3A). These peaks were annotated by comparing the accurate mass $(\mathrm{m} / \mathrm{z})$ and retention time with those provided in the National Institute of Standards and Technology online databases. The relative intensities of these metabolites were used in the subsequent multivariate statistical analysis. ${ }^{25}$

Table I Basic background data of NOD and CON macaques

\begin{tabular}{|l|l|l|l|}
\hline Variable & CON & NOD & -value \\
\hline Sample size $(\mathrm{n})$ & $\mathrm{I}$ & 10 & - \\
\hline Sex & $\mathrm{F}$ & F & - \\
\hline Age (years) & $12.50 \pm 0.66$ & $13.06 \pm 1.90$ & 0.475 \\
\hline Weight $(\mathrm{Kg})$ & $5.57 \pm 0.59$ & $5.93 \pm 0.88$ & 0.629 \\
\hline
\end{tabular}

Note: Data are presented as mean \pm SE.

Abbreviations: CON, control; F, female; NOD, naturally occurring depressive; SE, standard error. 


\section{Metabolomic data analysis}

The differential metabolites were identified based on the methods described previously. ${ }^{26-28}$ The peak indexes (RT-m/z), sample names and normalized peak intensities, were imported into SIMCA-P 14.0 (Umetrics, Umeå, Sweden) for multivariate statistical analyses. Multivariate statistical analyses, including principal component analysis (PCA) and pair-wise orthogonal projections to latent structures discriminant analyses (OPLS-DA) with Pareto scaling spectral data, were performed to visually discriminate samples in the NOD macaques and CON group. Three parameters $\left(R^{2} X, R^{2} Y\right.$, and $\left.Q^{2} Y\right)$ were used to quantify the quality of the model. $R^{2} X$ and $R^{2} Y$ were used to quantify the goodness-of-fit, and $\mathrm{Q}^{2} \mathrm{Y}$ was applied to assess the predictability of the model. If the $\mathrm{Q}^{2}$ and $\mathrm{R}^{2}$ values resulting from the original model were higher than the corresponding values from the permutation test, the model was considered valid. The candidate metabolites were selected according to thresholds of variable importance in the projection values (variable importance in the projection [VIP] $>1.0$ ) in the OPLS-DA model and a $P$-value $<0.05$ (obtained from two-tailed Student's $t$-test).

\section{Molecular pathway and network analysis}

The overall workflow involved in identifying the differential metabolites in NOD macaques is summarized in Figure 2. For that significantly changed metabolites (VIP $>1.0$ and $P<0.05$ ), pathway analysis was performed using MetaboAnalyst 3.0 (http://www.metaboanalyst.ca) ${ }^{29}$ and Ingenuity Pathways Analysis (IPA) software (Qiagen, NV, Venlo, the Netherlands). ${ }^{30}$ MetaboAnalyst was used to generate a heat map of all the different metabolites. To identify the predicted molecular pathways and to explore the biological functions of interested molecules, identified metabolites (with Human Metabolome Database [HMDB] identifiers) and corresponding fold changes were uploaded to the IPA server. The predicted molecular pathways and biological functions were generated based on the knowledge sorted in the IPA Knowledge Base. Cytoscape software (version 3.6.0) was used to build the correlation network between these differential metabolites in CSF and serum.

\section{Statistical analysis}

Statistical analyses between the two groups were performed using IBM SPSS Statistics for Windows, version 20.0 (IBM Corp., Armonk, NY, USA). Student's $t$-test was used for the data that examined normal distributions and equal variances, otherwise nonparametric Mann-Whitney U-test was used. A $P$-value $>0.05$ was considered statistically significant.

\section{Results}

\section{Significant differences in behaviors between NOD and CON groups}

The behavioral results were consistent with those reported by a previous study. ${ }^{16}$ Ten NOD macaques were selected based on our previous observations, ${ }^{16}$ and these animals displayed the highest frequency of depressive behaviors (Figure 1B). To match by age and sex, 12 healthy adult female $M$. fascicularis (aged 9-13 years) were selected (controls, CON) (Figure 1C). There were no significant differences in age or weight between the NOD macaques and CON group $(P>0.05)$.

The 53 discrete behaviors belonging to 12 behavioral categories were used to distinguish the NOD and CON groups. Eighteen behaviors pertaining to five behavioral categories were found to be significantly altered between NOD and CON macaques (Figure 1D). Six behaviors (licking tail, walking on the skylight, sitting on floor, feeding while hanging, scratching by hind leg, and huddling/embracing) were significantly increased in NOD group relative to the CON group, while 12 behaviors (walking on iron chain, walking on shelf, drinking, sitting on floor facing wall, nursing infant, standing, hanging on iron chain, scratching by foreleg, quadrupedal walking on floor, being groomed, grooming, and perching on shelf) were significantly reduced in NOD group relative to the CON group. The behavioral results indicated that the NOD group displayed NOD behaviors compared with the CON group.

\section{Metabolomics analysis and identification of significant metabolites from CSF samples of NOD and control macaque}

In total, the GC-MS metabolomics profiling for the CSF samples produced 663 variables across the NOD and CON groups, which were used in the subsequent multivariate analysis. Representative GC-MS TIC chromatograms are shown in Figure 3A. The PCA scores plot showed clear differences between the depressed and CON groups. This analysis showed that the NOD macaques were obviously discriminated from the healthy controls $\left(R^{2} X=0.754\right.$, $\mathrm{Q}^{2}=0.429$ ). The pair-wise PLS-DA score plots also revealed that the depressed macaques were statistically distinguishable from the CON group $\left(\mathrm{R}^{2} \mathrm{X}=0.802, \mathrm{R}^{2} \mathrm{Y}=0.936\right.$, and $\left.\mathrm{Q}^{2}=0.444\right)$. The three-dimensional diagram intuitively demonstrated clear separation between the NOD and CON 


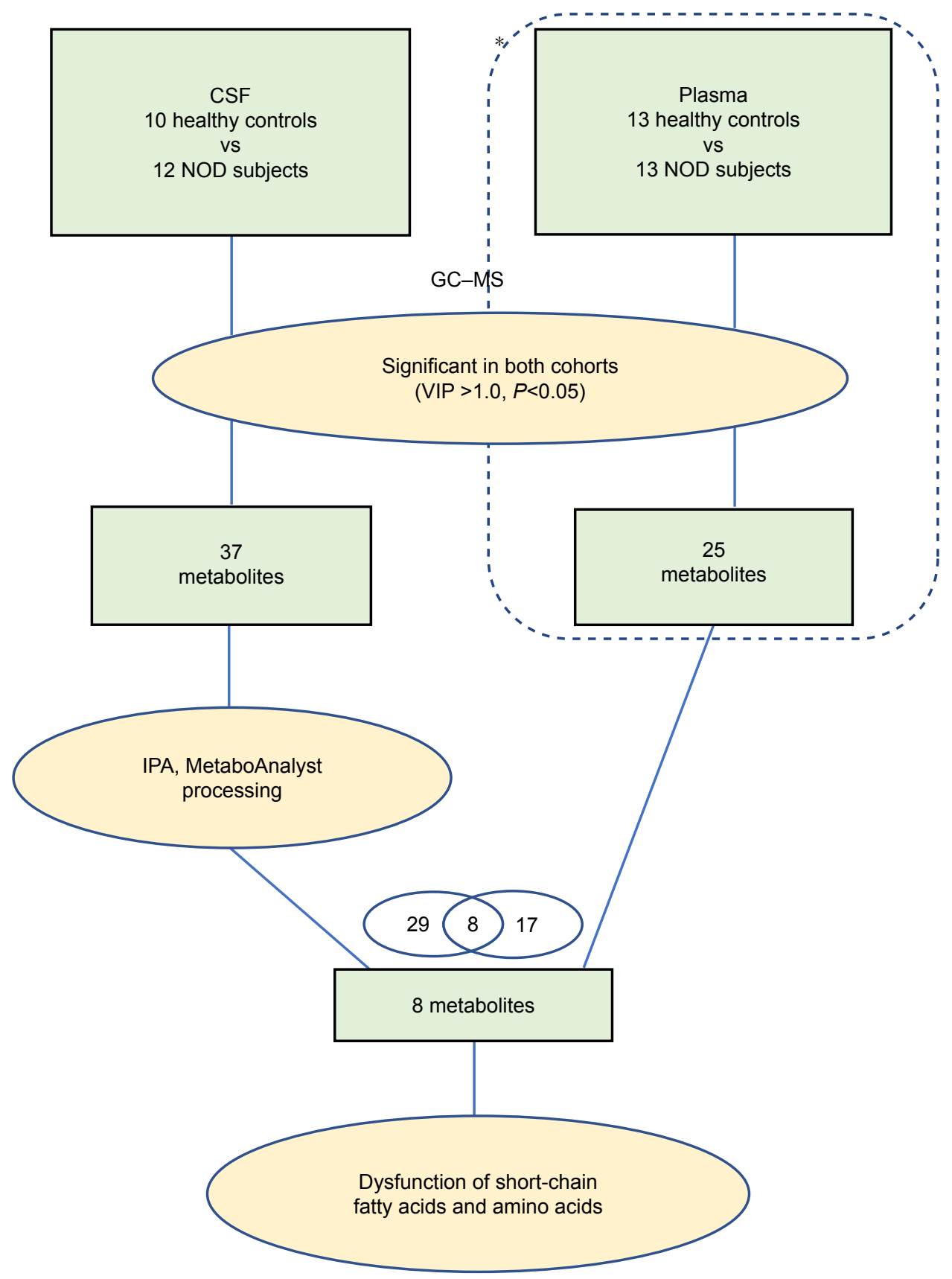

Figure 2 An overview of the GC-MS-based metabonomic workflow identifying differentially expressed CSF metabolites in NOD macaques. Note: *The data was from our published work $\left(X u\right.$ et al $\left.{ }^{16}\right)$.

Abbreviations: CON, control; NOD, naturally occurring depressive; GC-MS, gas chromatography-mass spectrometry; CSF, cerebrospinal fluid; VIP, variable importance in the projection; IPA, Ingenuity Pathways Analysis.

groups (Figure $3 \mathrm{~B}$ ). $\mathrm{R}^{2} \mathrm{X}$ and $\mathrm{R}^{2} \mathrm{Y}$ represent the cumulative model variations in $\mathrm{X}$ and $\mathrm{Y}$, respectively, and $\mathrm{Q}^{2}$ is the cumulative predicted variation. The values of these parameters approaching 1.0 indicate a stable model with predictive reliability.

According to the PLS-DA analysis, significant differential metabolites between the NOD group and the CON group were identified in the CSF samples. Using a VIP $>1.0$ in the PLS-DA models and $P<0.05$ using two-tailed Student's $t$-test, 37 differential metabolites were identified between the two groups (Table 2, Figure 4A). When compared to healthy controls, NOD macaques were characterized by higher levels of propanoic acid, acetic acid, hydroxylamine, propanedioic acid, butanoic acid, proline, methanamine, glycine, isothiourea, nonanoic acid, carbamic acid, threonine, 
A
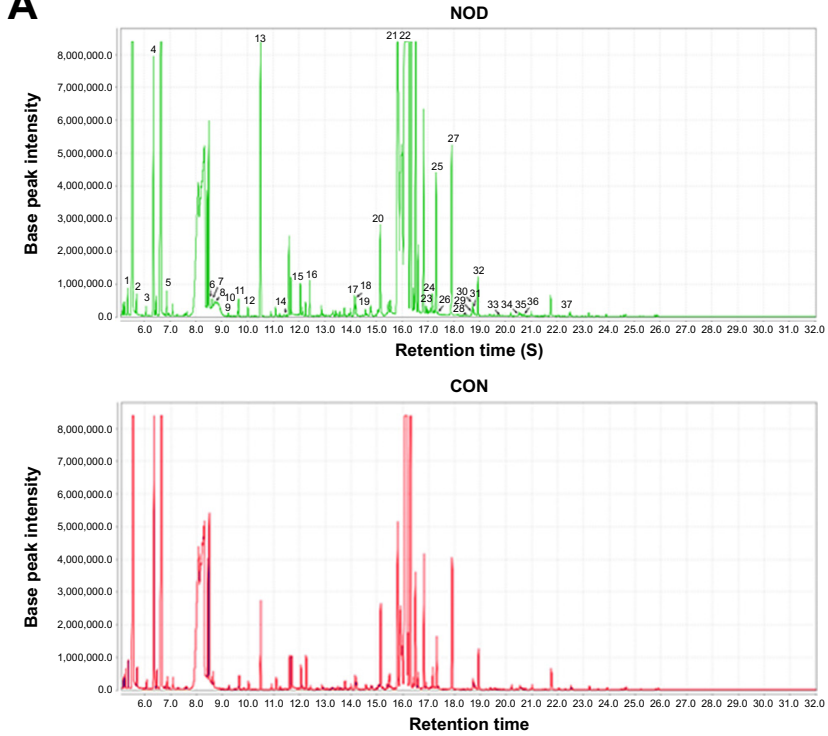

B

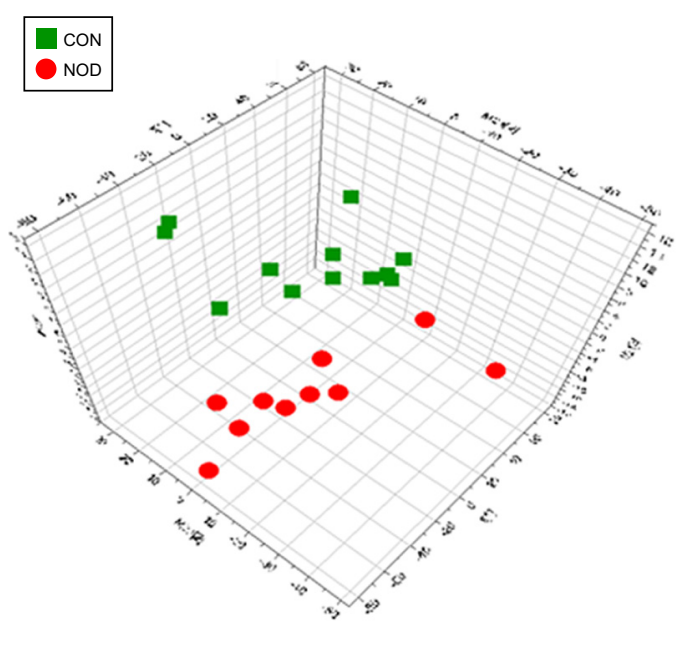

Figure 3 (A) Representative GC-MS total ion chromatograms (TIC) of the CON and NOD group. (B) Partial least squares-discriminate analysis (PLS-DA) scores plot derived from GC-MS spectra of the CON and NOD macaques.

Abbreviations: CON, control; NOD, naturally occurring depressive; GC-MS, gas chromatography-mass spectrometry.

beta-alanine, threitol, erythronic acid, L-aspartic acid, xylitol, ribitol, 2-keto-D-gluconic acid, 1,4-butanediamine, Dfructose, myoinositol, glucaric acid, hexadecanoic acid, scyllitol, gulose, heptadecanoic acid, linolelaidic acid, trans9-octadecenoic acid, oleic acid, octadecanoic acid, N-acetyl-Dglucosamine, D-glycero-D-galacto-heptitol, galactitol, 5-phenylvaleric acid, in addition to lower levels of benzeneacetic acid and $1 \mathrm{H}$-indole-2-carboxylic acid.

\section{Metabolic pathway and function analyses}

Using the HMDB classification, among the 37 metabolites, over $35.1 \%$ were sub-grouped as carbohydrates and carbohydrate conjugates; about $32.4 \%$ as lipids; and about $21.6 \%$ as amino acids, peptides, and analogues (Figure 4B). These metabolites were primarily located in cytoplasm $(29.7 \%)$, extracellular $(27.0 \%)$, membrane $(16.2 \%)$, mitochondria (10.8\%), and lysosome (8.1\%) (Figure 4C).

To understand the molecular functional roles of the differential metabolites, they were mapped into the IPA Knowledge Base. The NOD macaques were characterized by disturbances in the following top five canonical pathways: 1) fatty acid biosynthesis, 2) ABC transport system, 3) aminoacyl-tRNA biosynthesis, 4) aspartate metabolism, and 5) threonine degradation. The detailed results of pathway analysis are shown in Table 3. The differential metabolites found in NOD monkeys were predicted to be significantly involved in the biological functions like biosynthesis of lipids and uptake of amino acids (Figure 4D-E).

\section{Relationship between peripheral and central metabolic disturbances in NOD and control macaques}

To develop an integrated understanding of the central and peripheral metabolic disturbances that occurred in NOD macaques, we compared the differential metabolites identified in this study with those of our published findings. A correlation network was built to show the trend of metabolic disturbances in serum and CSF (Figure 5). Among these differential metabolites, eight metabolites (butanoic acid, 1-proline, 1-threonine, threitol, 2-keto-D-gluconic acid, 1,4-butanediamine, D-fructose, and myo-inositol) were altered both in the serum and CSF of NOD macaques. The eight altered metabolites were mainly identified as shortchain fatty acids and amino acids. These findings suggest that perturbations of short-chain fatty acid and amino acid metabolism in serum and CSF are implicated in the onset of depression.

\section{Discussion}

In this study, we for the first time characterized the metabolic changes of CSF samples from NOD cynomolgus monkeys relative to matched controls. We found that the NOD macaques were mainly characterized by perturbations in fatty acid biosynthesis, ABC transport system, and amino acid metabolism. Interestingly, the eight altered CSF metabolites, belonging to short-chain fatty acid and amino acids, were 
Table 2 Differential metabolites of cerebrospinal fluid (CSF) between NOD and CON macaques

\begin{tabular}{|c|c|c|c|c|c|c|c|}
\hline No & Metabolite & HMDB ID & Ret (min) & $\mathrm{m} / \mathrm{z}$ & VIP & $P$-value & $\log (F C)$ \\
\hline 1 & Propanoic acid & HMDB00237 & 5.3654 & 174 & 1.26 & 0.029 & 0.103 \\
\hline 2 & Acetic acid & HMDB00042 & 5.7046 & 205 & 1.32 & 0.022 & 0.137 \\
\hline 3 & Hydroxylamine & HMDB03338 & 6.2860 & 154 & 1.16 & 0.046 & 0.074 \\
\hline 4 & Propanedioic acid & HMDB0069I & 6.4586 & 218 & 1.15 & 0.049 & 0.089 \\
\hline 5 & Butanoic acid & HMDB00039 & 6.9491 & 145 & 1.36 & 0.017 & 0.143 \\
\hline 6 & L-proline & HMDB00I 62 & 8.7842 & 142 & 1.21 & 0.038 & 0.105 \\
\hline 7 & Methanamine & HMDB00I64 & 8.8266 & 160 & 1.33 & 0.021 & 0.086 \\
\hline 8 & Glycine & HMDB00I 23 & 8.9144 & 174 & 1.65 & 0.003 & 0.169 \\
\hline 9 & Isothiourea & HMDB34I55 & 9.3807 & 245 & 1.17 & 0.046 & 0.081 \\
\hline 10 & Nonanoic acid & HMDB00847 & 9.5321 & 215 & 1.32 & 0.021 & 0.108 \\
\hline 11 & Carbamic acid & HMDB0355I & 9.6229 & 278 & 1.38 & 0.016 & 0.142 \\
\hline 12 & Threonine & HMDB00I67 & 10.0166 & 117 & 1.33 & 0.020 & 0.097 \\
\hline 13 & Beta-alanine & HMDB00056 & 10.5041 & 73 & 1.51 & 0.007 & 0.165 \\
\hline 14 & Threitol & HMDB04I36 & 11.5125 & 217 & 1.76 & 0.001 & 0.155 \\
\hline 15 & Erythronic acid & HMDB006I3 & 12.0575 & 205 & 1.93 & 0.000 & 0.164 \\
\hline 16 & L-aspartic acid & HMDB00I9I & 12.4118 & 147 & 1.16 & 0.046 & 0.238 \\
\hline 17 & Xylitol & HMDB029I7 & 14.1409 & 103 & 1.59 & 0.004 & 0.146 \\
\hline 18 & Ribitol & HMDB00508 & 14.1984 & 117 & 1.19 & 0.040 & 0.116 \\
\hline 19 & 2-keto-D-gluconic acid & METPA0825 & 14.6496 & 292 & 1.29 & 0.024 & 0.126 \\
\hline 20 & I,4-butanediamine & HMDBOI4I4 & 15.3885 & 86 & 1.20 & 0.038 & 0.095 \\
\hline 21 & D-fructose & HMDB00660 & 15.7973 & 73 & 1.46 & 0.009 & 0.166 \\
\hline 22 & Myoinositol & HMDB002II & 16.0304 & 265 & 1.19 & 0.040 & 0.128 \\
\hline 23 & Glucaric acid & HMDB00663 & 17.0993 & 333 & 1.35 & 0.018 & 0.223 \\
\hline 24 & Hexadecanoic acid & HMDB00220 & 17.1599 & 73 & 1.21 & 0.038 & 0.086 \\
\hline 25 & Scyllitol & HMDB06088 & 17.2326 & 318 & 1.49 & 0.008 & 0.160 \\
\hline 26 & Gulose & HMDBI 2326 & 17.3052 & 204 & 1.38 & 0.016 & 0.219 \\
\hline 27 & Heptadecanoic acid & HMDB02259 & 18.0653 & 327 & 1.15 & 0.048 & 0.092 \\
\hline 28 & Benzeneacetic acid & HMDB00209 & 18.5165 & 295 & 1.21 & 0.037 & -0.055 \\
\hline 29 & Linolelaidic acid & HMDB06270 & 18.6800 & 337 & 1.37 & 0.016 & 0.117 \\
\hline 30 & Trans-9-octadecenoic acid & HMDB00573 & 18.7194 & 341 & 1.26 & 0.030 & 0.126 \\
\hline 31 & Oleic acid & HMDB00207 & 18.7799 & 68 & 1.17 & 0.044 & 0.104 \\
\hline 32 & Octadecanoic acid & HMDB00827 & 18.9374 & 257 & 1.17 & 0.044 & 0.085 \\
\hline 33 & IH-indole-2-carboxylic acid & HMDB02285 & 19.7853 & 369 & 1.29 & 0.025 & -0.051 \\
\hline 34 & $\mathrm{~N}$-acetyl-D-glucosamine & HMDB002I5 & 20.5362 & 73 & 2.05 & 0.000 & 0.176 \\
\hline 35 & D-glycero-D-galacto-heptitol & HMDB33750 & 20.5907 & 262 & 2.05 & 0.000 & 0.192 \\
\hline 36 & Galactitol & HMDB00I07 & 20.6634 & 307 & 1.87 & 0.000 & 0.191 \\
\hline 37 & 5-phenylvaleric acid & HMDB02043 & 22.3046 & 334 & 1.50 & 0.007 & 0.179 \\
\hline
\end{tabular}

Notes: Obtained from PLS-DA with a threshold of I.0. A positive $\log _{2}(\mathrm{FC})$ indicates significantly higher levels in NOD group compared with CON group. A negative log 2 (FC) indicates significantly lower levels in NOD group compared with CON group.

Abbreviations: VIP, variable importance in the projection; CON, control; NOD, naturally occurring depressive; Ret, retention time; FC, fold change; PLS-DA, partial least squares-discriminate analysis.

also observed in the serum sample of NOD model. These findings suggest that perturbations in peripheral and central short-chain fatty acids and amino acids are implicated in the onset of depression. Perturbations in lipid and amino acid metabolism were also reported in depressive patients ${ }^{31,32}$ and rodent models. ${ }^{6,33,34}$
Higher primates like cynomolgus monkeys appear to be particularly appropriate as a model for mental disorder. ${ }^{35}$ The cynomolgus monkey model described in our study better resembles the etiological development of depression compared to traditional rodent models, as these depressive monkeys were screened from an approximately natural 
A

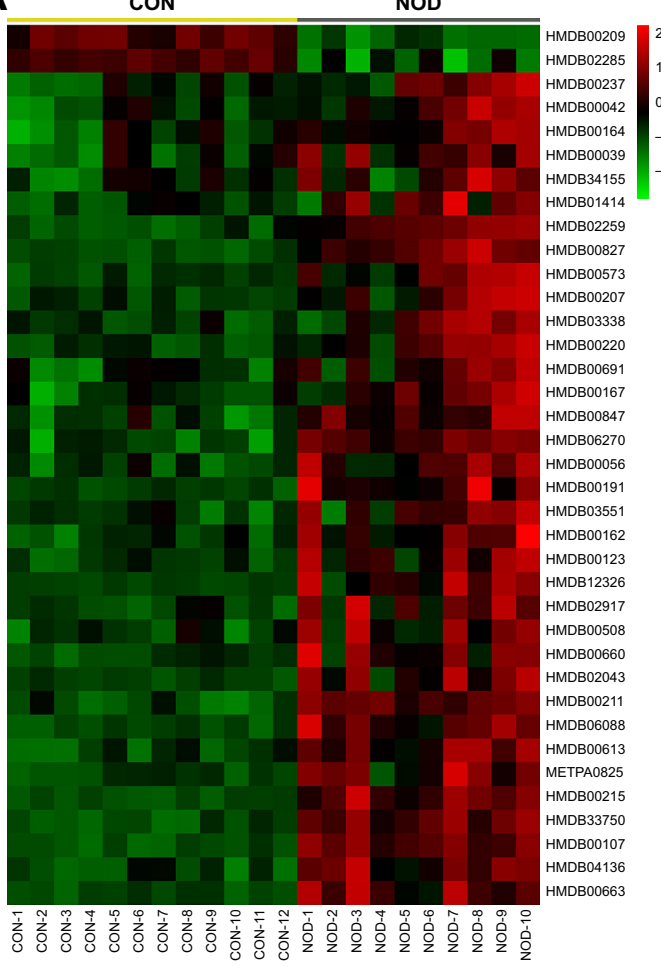

B
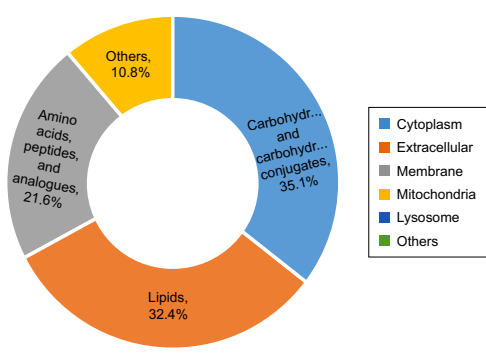

D

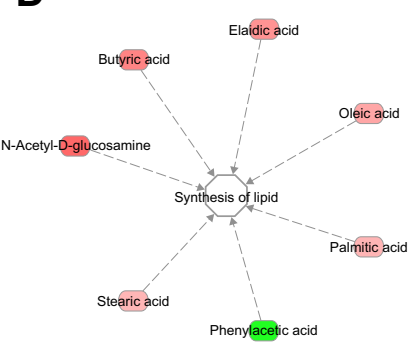

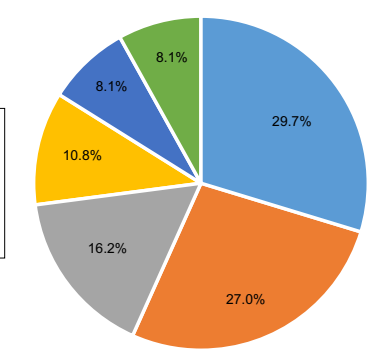

C

E

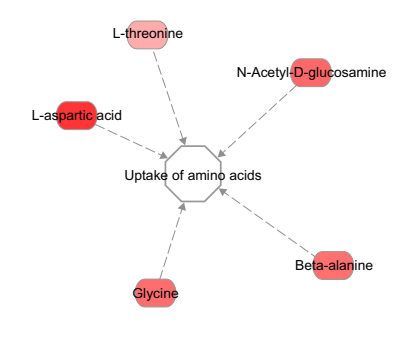

Figure 4 (A) Heat map of differentially expressed CSF metabolites in CON and NOD macaques. (B) Chemical taxonomy and (C) cellular locations based on the annotations of Human Metabolome Database (HMDB). The predicted biological functions were significantly involved in the biosynthesis of lipid (D) and uptake of amino acids (E). Abbreviations: CON, control; NOD, naturally occurring depressive; CSF, cerebrospinal fluid.

environment. In this condition, the depressed monkeys demonstrated superior validity without any human intervention, which may better imitate the psychosocial stressors encountered by adult humans living in a resource-based competitive society.

In this study, we found that fatty acid biosynthesis was significantly disturbed in CSF of NOD macaques relative to control. Fatty acids influence a number of cellular functions at different levels of incorporation, including cell membrane fluidity, membrane protein-mediated responses, eicosanoid generation, gene expression, and cell signaling. Through these mechanisms, fatty acids influence cell and tissue physiology, and the way cells and tissues respond to external signals in both the serum and CSF. ${ }^{36,37}$ Evidence suggests that in appropriate quantities, both saturated fatty acids and their derivatives are required for brain health. In a recent study ${ }^{38}$ using human frontal cortex tissue demonstrated that lipid rafts, membrane structures intimately associated with cell signalling, are predominately composed of the saturated fatty acids palmitic and stearic acid. Oleic acid, formed from the desaturation of stearic acid, has been shown to promote axonogenesis in the striatum during brain development, ${ }^{39}$ and is used as a cerebral energy source when glucose availability diminishes. Consistent with these studies, our

Table 3 Top five affected ingenuity canonical pathways enriched in CSF between NOD and CON macaques

\begin{tabular}{|l|l|l|l|l|l|}
\hline Pathway name & Total & Hits & P-value & FDR & Matching IDs \\
\hline Fatty acid biosynthesis & 16 & 6 & $2.98 \mathrm{e}-06$ & $1.01 \mathrm{e}-04$ & $\begin{array}{l}\text { HMDB00042 HMDB0069I HMDB00039 } \\
\text { HMDB00220 HMDB00207 HMDB00827 }\end{array}$ \\
\hline ABC transporters & 22 & 5 & $5.43 \mathrm{e}-05$ & $1.36 \mathrm{e}-03$ & $\begin{array}{l}\text { HMDB00I23 HMDB00I67 HMDB00I9I } \\
\text { HMDB0I4I4 HMDB002I5 }\end{array}$ \\
\hline Aminoacyl-tRNA biosynthesis & 20 & 4 & $1.63 \mathrm{e}-04$ & $5.22 \mathrm{e}-03$ & $\begin{array}{l}\text { HMDB00I62 HMDB00I23 HMDB00I67 } \\
\text { HMDB00I9I }\end{array}$ \\
\hline Aspartate metabolism & 16 & 3 & $5.69 \mathrm{e}-04$ & $9.67 \mathrm{e}-03$ & HMDB0069I HMDB00056 HMDB00I9I \\
\hline Threonine degradation & 17 & 3 & $1.69 \mathrm{e}-04$ & $1.46 \mathrm{e}-02$ & HMDB00237 HMDB00I23 HMDB00I67 \\
\hline
\end{tabular}

Abbreviations: CON, control; NOD, naturally occurring depressive; CSF, cerebrospinal fluid; FDR, false discovery rate. 


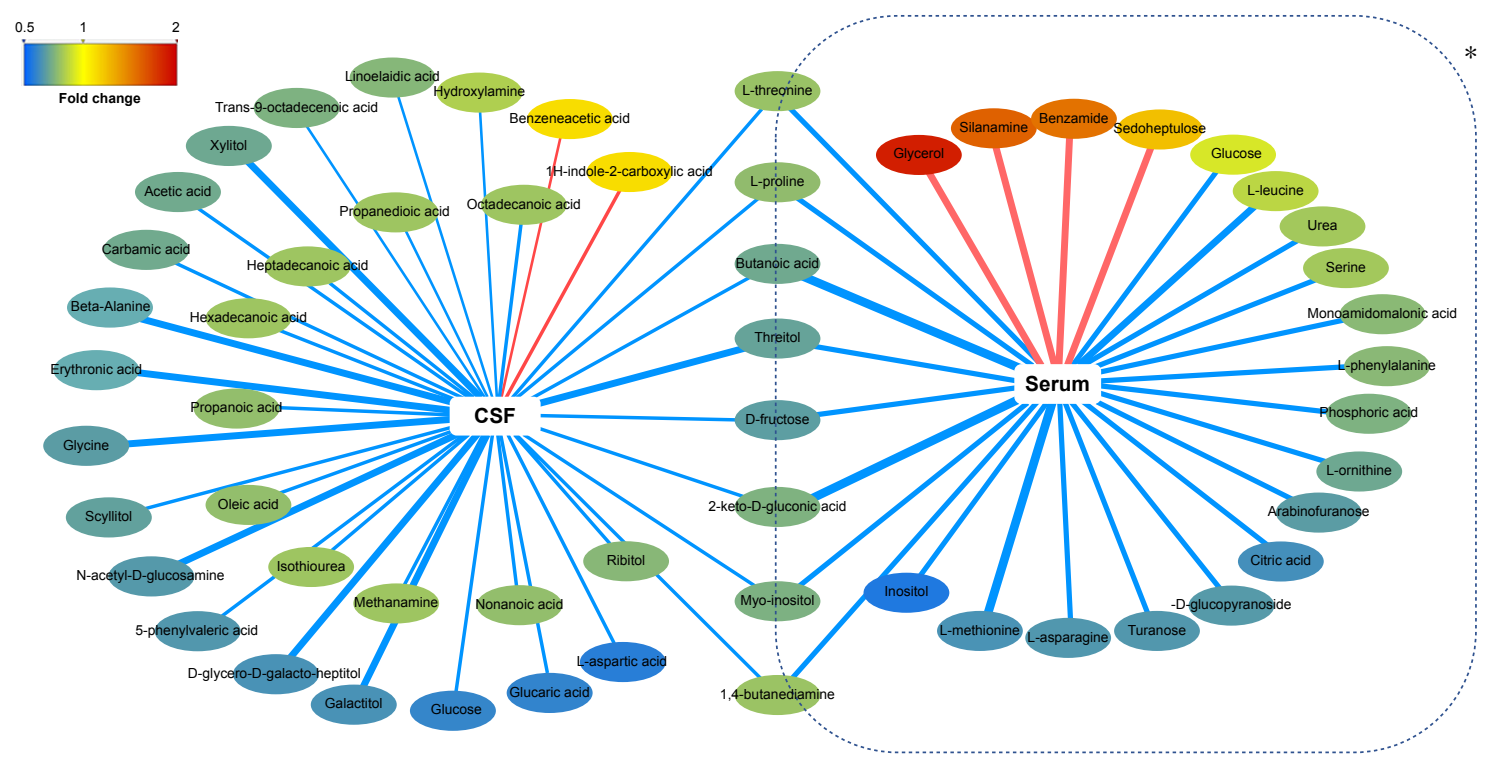

Figure 5 Network analysis of differentially expressed metabolites in CSF and serum of NOD macaques.

Notes: *The data was from our published work $\left(\mathrm{Xu}\right.$ et $\left.\mathrm{al}^{16}\right)$. Red-colored boxes and red-colored lines indicate upregulation in NOD macaques compared with control $(\mathrm{CON})$, while green-colored boxes and green-colored lines indicate downregulation. The width of the line represents the VIP scores of differential metabolites. Abbreviations: NOD, naturally occurring depressive; CSF, cerebrospinal fluid; VIP, variable importance in the projection.

results indicated that oleic acid, octadecanoic acid, and hexadecanoic acid were markedly perturbed in NOD macaques compared with control. In addition, short-chain fatty acids (acetic acid, propanedioic acid, and butyric acid) were also found to be disturbed in CSF of NOD macaques. These short-chain fatty acids are important metabolites for the gut microbiota, which are important for host health, as they provide the preferential energy source for intestinal epithelium, stimulate the production of regulatory $\mathrm{T}$ cells, inhibit inflammation, and regulate gene expression as a histone deacetylase inhibitor. ${ }^{40}$ The entire amount of butyrate that our body needs is produced by butyrate-producing bacteria living in our gut. ${ }^{41}$ In the present study, butyrate level was found to be significantly different both in the serum and CSF samples of NOD macaques.

In addition to the reduced short-chain fatty acids, two microbiotic metabolites - methanamine and benzeneacetic acid - were decreased in NOD macaques relative to HCs. These metabolites are uniquely produced by microbial metabolism in the intestinal tract, indicating that major depressive disorder (MDD) may be associated with variations in intestinal microflora. Consistent with this result, our previous studies $^{3,42}$ showed that several altered metabolites in MDD macaques are the metabolic byproducts of gut microbiota. This is the first study to detect microbial-specific metabolites both in the periphery and brain of the same non-human primate model of depression. These findings may contribute to a better understanding of the microbiota-gut-brain axis.
$\mathrm{ABC}$ transporters, which are important components of the blood-brain barrier (BBB), function by either preventing the entry of or expelling numerous molecules including toxins, drugs, and other xenobiotics. ${ }^{43}$ In the present study, five differential metabolites, involved in $\mathrm{ABC}$ transport system, were observed in NOD macaques. The disturbed $\mathrm{ABC}$ transport system may affect BBB permeability, leading to some neuropsychiatric disorders. ${ }^{44}$ It has been shown that various physical or psychological stressors can impact the BBB's ability to selectively restrict the passage of substances from the blood to the brain. ${ }^{45}$ Deranged BBB permeability has been reported in MDD which implies that protein exchange may occur between the brain and peripheral circulation. The ABC transporters have been recognized to play an important role in neurological diseases like Alzheimer's and Parkinson's, depression, schizophrenia, and epilepsy. ${ }^{46}$ Some studies suggested that $A B C$ transporters family and their genetic polymorphisms may play a role in mediating antidepressant response. ${ }^{47,48}$

Aminoacyl-tRNA biosynthesis and a panel of amino acids were significantly disturbed in CSF of NOD macaques compared to HCs. The aminoacyl-tRNA synthetases are prominently known for their classic function in the first step of protein synthesis, where they bear the responsibility of setting the genetic code. ${ }^{49}$ Disturbed amino acid metabolism was regarded as an important factor in depression. Some clinical evidence ${ }^{50,51}$ suggested that the plasma levels of aspartic acid and glycine were significantly downregulated 
in depression macaques. In animal models of depression, proline was also found to be remarkably decreased. ${ }^{52}$ Consistent with these studies, our results showed that the levels of proline, glycine, threonine, and aspartic acid decreased in NOD macaques, with perturbed pathway of aspartate metabolism; threonine degradation; glycine, serine, and threonine metabolism; and arginine and proline metabolism. Our published studies also found that disturbance in the metabolism of certain amino acids was an important factor contributing to MDD in serum and CSF. 5,6,34,42,53

\section{Limitations}

There are some limitations in this study that should be recognized. Firstly, the absolute value of our sample size was not particularly large because of the high cost and ethical regulations involved in the use of monkeys for animal experiments. Secondly, only female NOD macaques were employed. It is required to determine whether the altered metabolites exhibit gender specificity. Thirdly, we have no results with regard to metabolic changes in the brain. Further studies that can capture the metabolic changes in key brain regions of depressive monkeys are required.

\section{Conclusion}

Employing a GC-MS metabolomic approach, we found that metabolic profiling of CSF sample from NOD macaques was significantly different from that of $\mathrm{CON}$ macaques. The NOD group was mainly associated with disturbances in fatty acid biosynthesis, ABC transport system, and amino acid metabolism. Moreover, some CSF metabolites belonging to short-chain fatty acids and amino acids were found to be changed in the serum of NOD model. Our findings suggest that changes in peripheral and central short-chain fatty acid and amino acid metabolism are implicated in the onset of depression.

\section{Acknowledgments}

This work was supported by the National Key R\&D Program of China (Grant Nos 2017YFA0505700 and 2016YFC1307200), the National Natural Science Foundation of China (Grant Nos 81701360 and 81701361), Chongqing Post-Doctoral Science Foundation Projects (Grant No Xm2017026), and Chongqing Science \& Technology Commission (cxth2017jcyjA0207).

\section{Disclosure}

The authors report no conflicts of interest in this work.

\section{References}

1. WHO. Depression. 2017. Available from: http://www.who.int/mediacentre/factsheets/fs369/en/Accessed March 22, 2018.

2. Novick D, Montgomery W, Aguado J, Peng X, Haro JM. Impact of anxiety symptoms on outcomes of depression: an observational study in Asian patients. Neuropsychiatr Dis Treat. 2016;12:795-800. doi:10. 2147/NDT.S90134

3. Zheng P, Zeng B, Zhou C, et al. Gut microbiome remodeling induces depressive-like behaviors through a pathway mediated by the host's metabolism. Mol Psychiatry. 2016;21(6):786-796. doi:10.1038/ mp. 2016.44

4. Eberhard J, Weiller E. Suicidality and symptoms of anxiety, irritability, and agitation in patients experiencing manic episodes with depressive symptoms: a naturalistic study. Neuropsychiatr Dis Treat. 2016;12: 2265-2271. doi:10.2147/NDT.S111094

5. Zhou X, Liu L, Zhang Y, et al. Metabolomics identifies perturbations in amino acid metabolism in the prefrontal cortex of the learned helplessness rat model of depression. Neuroscience. 2017;343:1-9. doi:10. 1016/j.neuroscience.2016.11.038

6. Wu Y, Fu Y, Rao C, et al. Metabolomic analysis reveals metabolic disturbances in the prefrontal cortex of the lipopolysaccharide-induced mouse model of depression. Behav Brain Res. 2016;308:115-127. doi:10.1016/j.bbr.2016.04.032

7. Wang W, Guo H, Zhang S-X, et al. Targeted metabolomic pathway analysis and validation revealed glutamatergic disorder in the prefrontal cortex among the chronic social defeat stress mice model of depression. J Proteome Res. 2016;15(10):3784-3792. doi:10.1021/ acs.jproteome.6b00577

8. Pan J, Liu H, Zhou J, et al. Ipsilateral hippocampal proteomics reveals mitochondrial antioxidative stress impairment in cortical-lesioned chronic mild stressed rats. Curr Mol Med. 2014;14(9):1186-1196.

9. Hu Q, Shen P, Bai S, et al. Metabolite-related antidepressant action of diterpene ginkgolides in the prefrontal cortex. Neuropsychiatr Dis Treat. 2018;14:999-1011. doi:10.2147/NDT.S161351

10. Bianchi R, Schonfeld IS, Vandel P, Laurent E. On the depressive nature of the "burnout syndrome": A clarification. Eur psychiatry. 2017;41: 109-110. doi:10.1016/j.eurpsy.2016.10.008

11. Yoshimi N, Futamura T, Bergen SE, et al. Cerebrospinal fluid metabolomics identifies a key role of isocitrate dehydrogenase in bipolar disorder: evidence in support of mitochondrial dysfunction hypothesis. Mol Psychiatry. 2016;21(11):1504-1510. doi:10.1038/mp.2015.217

12. Hestad KA, Engedal K, Whist JE, et al. Patients with depression display cytokine levels in serum and cerebrospinal fluid similar to patients with diffuse neurological symptoms without a defined diagnosis. Neuropsychiatr Dis Treat. 2016;12:817-822. doi:10.2147/NDT.S101925

13. Sinclair AJ, Viant MR, Ball AK, et al. NMR-based metabolomic analysis of cerebrospinal fluid and serum in neurological diseases-a diagnostic tool? NMR Biomed. 2010;23(2):123-132. doi:10.1002/nbm.1428

14. Ohman A, Forsgren L. NMR metabonomics of cerebrospinal fluid distinguishes between Parkinson's disease and controls. Neurosci Lett. 2015;594:36-39. doi:10.1016/j.neulet.2015.03.051

15. Kaddurah-Daouk R, Yuan P, Boyle SH, et al. Cerebrospinal fluid metabolome in mood disorders-remission state has a unique metabolic profile. Sci Rep. 2012;2:667. doi:10.1038/srep00386

16. Xu F, Wu Q, Xie L, et al. Macaques exhibit a naturally-occurring depression similar to humans. Sci Rep. 2015;5:9220. doi:10.1038/srep09220

17. Xie L, Zhou Q, Liu S, et al. Effect of living conditions on biochemi$\mathrm{cal}$ and hematological parameters of the cynomolgus monkey. $\mathrm{Am} \mathrm{J}$ Primatol. 2014;76(11):1011-1024. doi:10.1002/ajp.22285

18. Xu F, Xie L, Li X, et al. Construction and validation of a systematic ethogram of Macaca fascicularis in a free enclosure. PLoS One. 2012; 7(5):e37486. doi:10.1371/journal.pone.0037486

19. Xie L, Xu F, Liu S, et al. Age- and sex-based hematological and biochemical parameters for Macaca fascicularis. PLoS One. 2013;8(6): e64892. doi:10.1371/journal.pone.0064892 
20. Li X, Xu F, Xie L, et al. Depression-like behavioral phenotypes by social and social plus visual isolation in the adult female MACACA fascicularis. PLoS One. 2013;8(9):e73293. doi:10.1371/journal.pone.0073293

21. National Research Council Committee for the Update of the Guide for the $\mathrm{C}$, Use of Laboratory A. The National Academies Collection: reports funded by National Institutes of Health. In: Guide for the Care and Use of Laboratory Animals. Washington (DC): National Academies Press (US) National Academy of Sciences; 2011;182-183.

22. Xie L, Zhou Q, Liu S, et al. Normal thoracic radiographic appearance of the cynomolgus monkey (macaca fascicularis). PLoS One. 2014;9(1): e84599. doi:10.1371/journal.pone.0084599

23. Li X, Han P, Guo Y, Sun H, Xiao Y, Kang YJ. An improved technique for cerebrospinal fluid collection of cisterna magna in Rhesus monkeys. J Neurosci Methods. 2015;249:59-65. doi:10.1016/j.jneumeth.2015. 04.004

24. Coplan JD, Karim A, Chandra P, St Germain G, Abdallah CG, Altemus M. Neurobiology of maternal stress: role of social rank and central oxytocin in hypothalamic-pituitary adrenal axis modulation. Front Psychiatry. 2015;6:100. doi:10.3389/fpsyt.2015.00100

25. Luedemann A, Strassburg K, Erban A, Kopka J. TagFinder for the quantitative analysis of gas chromatography-mass spectrometry (GC-MS)-based metabolite profiling experiments. Bioinformatics. 2008; 24(5):732-737. doi:10.1093/bioinformatics/btn023

26. Chen J-J, Zhou C-J, Liu Z, et al. Divergent urinary metabolic phenotypes between major depressive disorder and bipolar disorder identified by a combined GC-MS and NMR spectroscopic metabonomic approach. J Proteome Res. 2015;14(8):3382-3389. doi:10.1021/acs.jproteome. 5 b00434

27. Zhang Y, Zhou X, Yang L, et al. Comparative efficacy and acceptability of psychotherapies for post-traumatic stress disorder in children and adolescents: study protocol for a systematic review and network meta-analysis. BMJ Open. 2018;8(3):e020198. doi:10.1136/bmjopen2017-020198

28. Chen Z, Bai S, Hu Q, et al. Ginkgo biloba extract and its diterpene ginkgolide constituents ameliorate the metabolic disturbances caused by recombinant tissue plasminogen activator in rat prefrontal cortex. $\mathrm{Neu}$ ropsychiatr Dis Treat. 2018;14:1755-1772. doi:10.2147/NDT.S167448

29. Xia J, Wishart DS. Using metaboanalyst 3.0 for comprehensive metabolomics data analysis. Current Protoc Bioinf. 2016;55:14.10.11-14.10.91. doi:10.1002/cpbi.11

30. Kramer A, Green J, Pollard J Jr, Tugendreich S. Causal analysis approaches in ingenuity pathway analysis. Bioinformatics. 2014;30(4): 523-530. doi:10.1093/bioinformatics/btt703

31. Xu H-B, Zhang R-F, Luo D, et al. Comparative proteomic analysis of plasma from major depressive patients: identification of proteins associated with lipid metabolism and immunoregulation. Inter JNeuropsychopharmacol. 2012;15(10):1413-1425. doi:10.1017/S1461145712000302

32. Xu H-B, Fang L, Hu Z-C, et al. Potential clinical utility of plasma amino acid profiling in the detection of major depressive disorder. Psychiatry Res. 2012;200(2-3):1054-1057. doi:10.1016/j.psychres.2012.05.027

33. Liang Z, Bai S, Shen P, et al. GC-MS-based metabolomic study on the antidepressant-like effects of diterpene ginkgolides in mouse hippocampus. Behav Brain Res. 2016;314:116-124. doi:10.1016/j.bbr.2016.08.001

34. Chen G, Yang D, Yang Y, et al. Amino acid metabolic dysfunction revealed in the prefrontal cortex of a rat model of depression. Behav Brain Res. 2015;278:286-292. doi:10.1016/j.bbr.2014.05.027

35. Arnsten AF, Wang M, Paspalas CD. Dopamine's actions in primate prefrontal cortex: challenges for treating cognitive disorders. Pharmacol Rev. 2015;67(3):681-696. doi:10.1124/pr.115.010512

36. Guest J, Garg M, Bilgin A, Grant R. Relationship between central and peripheral fatty acids in humans. Lipids Health Dis. 2013;12:79. doi:10. 1186/1476-511X-12-79
37. Cheng Y-S, Tseng P-T, Chen Y-W, et al. Supplementation of omega 3 fatty acids may improve hyperactivity, lethargy, and stereotypy in children with autism spectrum disorders: a meta-analysis of randomized controlled trials. Neuropsychiatr Dis Treat. 2017;13:2531-2543. doi:10.2147/NDT.S147305

38. Martin V, Fabelo N, Santpere G, et al. Lipid alterations in lipid rafts from Alzheimer's disease human brain cortex. J Alzheimers Dis. 2010; 19(2):489-502. doi:10.3233/JAD-2010-1242

39. Polo-Hernandez E, De Castro F, Garcia-Garcia AG, Tabernero A, Medina JM. Oleic acid synthesized in the periventricular zone promotes axonogenesis in the striatum during brain development. J Neurochem. 2010;114(6):1756-1766. doi:10.1111/j.1471-4159.2010.06891.x

40. Zhang Q, Wu Y, Wang J, et al. Accelerated dysbiosis of gut microbiota during aggravation of DSS-induced colitis by a butyrate-producing bacterium. Sci Rep. 2016;6:27572. doi:10.1038/srep27572

41. Pryde SE, Duncan SH, Hold GL, Stewart CS, Flint HJ. The microbiology of butyrate formation in the human colon. FEMS Microbiol Lett. 2002;217(2):133-139. doi:10.1111/j.1574-6968.2002.tb11467.x

42. Zheng $P$, Wang $Y$, Chen $L$, et al. Identification and validation of urinary metabolite biomarkers for major depressive disorder. MMol Cell Proteomics. 2013;12(1):207-214. doi:10.1074/mcp.M112.021816

43. Saunders NR, Habgood MD, Mollgard K, Dziegielewska KM. The biological significance of brain barrier mechanisms: help or hindrance in drug delivery to the central nervous system? F1000Research. 2016;5. doi:10.12688/f1000research.7378.1

44. Liu Q, Hou J, Chen X, et al. P-glycoprotein mediated efflux limits the transport of the novel anti-Parkinson's disease candidate drug FLZ across the physiological and PD pathological in vitro BBB models. PLoS One. 2014;9(7):e102442. doi:10.1371/journal.pone.0102442

45. Lochhead JJ, Ronaldson PT, Davis TP. Hypoxic stress and inflammatory pain disrupt blood-brain barrier tight junctions: implications for drug delivery to the central nervous system. Aaps J. 2017;19(4):910-920. doi:10.1208/s12248-017-0076-6

46. Benadiba M, Maor Y. Importance of ABC transporters in drug development. Curr Pharm Des. 2016;22(38):5817-5829.

47. Powell TR, Tansey KE, Breen G, et al. ATP-binding cassette sub-family F member 1 (ABCF1) is identified as a putative therapeutic target of escitalopram in the inflammatory cytokine pathway. J psychopharmacol. 2013;27(7):609-615. doi:10.1177/0269881113490329

48. Huang X, Yu T, Li X, et al. ABCB6, ABCB1 and ABCG1 genetic polymorphisms and antidepressant response of SSRIs in Chinese depressive patients. Pharmacogenomics. 2013;14(14):1723-1730. doi:10.2217/ pgs.13.151

49. Pang YL, Poruri K, Martinis SA. tRNA synthetase: tRNA aminoacylation and beyond. Wiley Interdiscip Rev RNA. 2014;5(4):461-480. doi:10.1002/wrna.1224

50. Lu Y-R, Fu X-Y, Shi L-G, et al. Decreased plasma neuroactive amino acids and increased nitric oxide levels in melancholic major depressive disorder. BMC Psychiatry. 2014;14:123. doi:10.1186/1471244X-14-123

51. Frye MA, Tsai GE, Huggins T, Coyle JT, Post RM. Low cerebrospinal fluid glutamate and glycine in refractory affective disorder. Biol Psychiatry. 2007;61(2):162-166. doi:10.1016/j.biopsych.2006.01.024

52. Shi B, Tian J, Xiang H, et al. A ${ }^{1} \mathrm{H}-\mathrm{NMR}$ plasma metabonomic study of acute and chronic stress models of depression in rats. Behav Brain Res. 2013;241:86-91. doi:10.1016/j.bbr.2012.11.036

53. Zheng P, Fang Z, Xu X-J, et al. Metabolite signature for diagnosing major depressive disorder in peripheral blood mononuclear cells. J Affect Disord. 2016;195:75-81. doi:10.1016/j.jad.2016.02.008 


\section{Supplementary material}

Table SI The included behaviors of 12 behavior categories

\begin{tabular}{|c|c|}
\hline Behavioral categories & Behavioral items \\
\hline Ingestion behaviors & $\begin{array}{l}\text { Searching, feeding while squatting, feeding while hanging, feeding while sitting, drinking, chewing, licking } \\
\text { residue from floor, picking remaining food, feeding while perched, suckling }\end{array}$ \\
\hline Thermo-regulatory behaviors & Huddling, quivering, embracing \\
\hline Rutting and estrous behaviors & $\begin{array}{l}\text { Licking genital area, presenting buttocks, peri-vulvar discoloration, sniffing anogenital area, sniffing urine, } \\
\text { rolling tongue, homosexual roaring, homosexual mounting, tail arching }\end{array}$ \\
\hline Mating behaviors & Mounting, copulating, post-copulation guarding, post-copulation standing, ejaculating, masturbating \\
\hline Resting behaviors & $\begin{array}{l}\text { Sitting on floor, sitting on floor facing wall, perching on shelf (socially dominant), sitting on ring, lying on } \\
\text { floor, lying on shelf, hanging on window or door (non-socially dominant), hanging on skylight, hanging on } \\
\text { iron chain, hanging on ventilator, sitting and sleeping }\end{array}$ \\
\hline Parental behaviors & Nursing infant, holding infant, defending infant, licking anus of infant, checking anus of infant \\
\hline Amicable behaviors & Grooming, being groomed, embracing \\
\hline Conflict behaviors & $\begin{array}{l}\text { Driving (socially dominant, aggressive), attacking (socially dominant, aggressive), fleeing (non-socially } \\
\text { dominant), pulling foreleg, pulling hind leg, protracting ears, threatening (socially dominant, aggressive), } \\
\text { being threatened (non-socially dominant), being attacked (non-socially dominant), parallel pacing, biting } \\
\text { (socially dominant, aggressive) }\end{array}$ \\
\hline Vigilance behaviors & Shifting position, alarmed jumping, watching company, alarmed calling, miscellaneous calling, shaking cage \\
\hline Locomotion behaviors & $\begin{array}{l}\text { Galloping, walking on shelf (socially dominant), quadrupedal walking on floor (non-socially dominant), } \\
\text { moving, climbing, walking on iron chain, walking on skylight, standing, stepping, trotting }\end{array}$ \\
\hline Communication behaviors & Lip smacking, sniffing, voiding, alarmed calling, miscellaneous calling \\
\hline Miscellaneous behaviors & $\begin{array}{l}\text { Huddling (solitary), shaking body, playing (solitary), licking hair, scratching by hind leg, scratching by foreleg, } \\
\text { yawning, licking hand, catching pest, digging anus, rubbing palm on floor, licking tail, shaking ID card }\end{array}$ \\
\hline
\end{tabular}

Neuropsychiatric Disease and Treatment

Dovepress

\section{Publish your work in this journal}

Neuropsychiatric Disease and Treatment is an international, peerreviewed journal of clinical therapeutics and pharmacology focusing on concise rapid reporting of clinical or pre-clinical studies on a range of neuropsychiatric and neurological disorders. This journal is indexed on PubMed Central, the 'PsycINFO' database and CAS, and is the official journal of The International Neuropsychiatric Association (INA). The manuscript management system is completely online and includes a very quick and fair peer-review system, which is all easy to use. Visit http://www.dovepress.com/testimonials.php to read real quotes from published authors. 